\title{
Electron tomography of tungsten tips by coherent and incoherent imaging modes of TEM/STEM
}

\author{
X. Xu*, Z. Saghi*, Y. Peng****, R. Gay****, B. J. Inkson*, and G. Möbus* \\ *Department of Engineering Materials \\ **Department of Electronic and Electrical Engineering \\ University of Sheffield, Sir Robert Hadfield Building, Mappin Street, Sheffield, S1 3JD, UK
}

Tungsten tips are commonly used for scanning tunneling microsocopy (STM). Recently, they have been used as manipulation probes to measure electrical and mechanical performance of nanomaterials in transmission electron microscopy (TEM) [1-3]. As W tips directly contact samples during measurement, their three-dimensional morphology and composition before and after testing are essential information and have to be well characterized. In this paper, $\mathrm{W}$ tips were imaged by bright field (BF), annular dark field (ADF), energy-filtered TEM (EFTEM), and energy dispersive X-ray spectroscopy (EDX). Their three-dimensional structures were reconstructed by electron tomography [4] using coherent and incoherent imaging modes [5-6]. In addition, we explored application limits of ADF-STEM and EDX for thickness (projection) mapping.

W tips were prepared by the electrochemical etching method. In order to verify later generated tomograms, we picked up some nanoobjects as reference markers by dipping the W tips into an aqueous solution of gold nanoparticles. Figure 1(a) shows a BF image of a typical W tip with some gold particles attracted to its surface by Van der Waals force. Two sets of tilt series of the same tip were recorded by BF and ADF-STEM across the range $-60^{\circ}$ to $+60^{\circ}$ at $5^{\circ}$ angular increment in a 2010F TEM (Jeol, Japan) at $200 \mathrm{kV}$. The ADF image at $0^{\circ}$ tilt angle is shown in Figure 1(b). Carbon contaminants were found growing on tip surface after a period of observation. Oxygen mapping shown in Figure 1(c) was obtained via the three-window method using GIF (Gatan, USA). A layer of tungsten oxide produced during the etching procedure was found covering the tip surface.

The BF and ADF-STEM tilt series were aligned and reconstructed using IMOD [4]. The generated tomograms are shown in Figure 2(a) and (b) by Interactive Data Language IDL (Research Systems, USA). The inner tungsten cone is highlighted in red color and it is covered by an outer layer of oxide in green. The carbon contaminants are shown in blue. A core/shell object consisting of amorphous layers (oxide and carbon) on crystalline core (tungsten, also gold markers) is an ideal test object to evaluate incoherent vs coherent imaging modes in tomography (see also [6,7]). We confirm that both methods are complementary. BF, providing better resolution than ADF, recovers the outer shape of the tip with little disturbance from the false projections of the crystalline core. The core itself is not well restored. The reproduction of the Au particles in BF, are visible but not as clear as $\mathrm{ADF}$, indicating a trade-off between higher resolution and worse projection accuracy.

As a second topic of discussion, significant artefacts due to the missing wedge will be illustrated by means of animated video sequences of the tomograms in various viewing directions. Finally in Fig 3 we illustrate the upper thickness limit for tomography in STEM, by means of nonlinear (and nonmonotonous) intensity- thickness relations (absorption, see also [8]). W-cones are again ideal test objects. EDX mapping tolerates the largest thickness, while the ADF limit depends on ADF parameters and A/D converter settings but is intermediate between EDX and EFTEM [9]. 
References:

1. T. Kuzumaki et al., Carbon 42 (2004) 2329.

2. M.Larsson et al., Microsc. Microanal. 10 (2004) 41.

3. M. S. Bobji et al., J. Mater. Res. 20 (2005) 2726.

4. J.R. Kremer et al., J. Struct. Biol. 116 (1996) 71.

5. G. Möbus and B. J. Inkson, Appl. Phy. Lett. 79 (2001) 1369 and Ultramicroscopy 96 (2003) 433.

6. P. A. Midgley and M. Weyland, Ultramicroscopy 96 (2003) 413.

7. H. Friedrich et al., Ultramicroscopy 106 (2005) 18.

8. S. Hillyard and J. Silcox, Ultramicroscopy 52 (1993) 325.

9. This work was supported by a grant from EPSRC, RA/104868, UK.

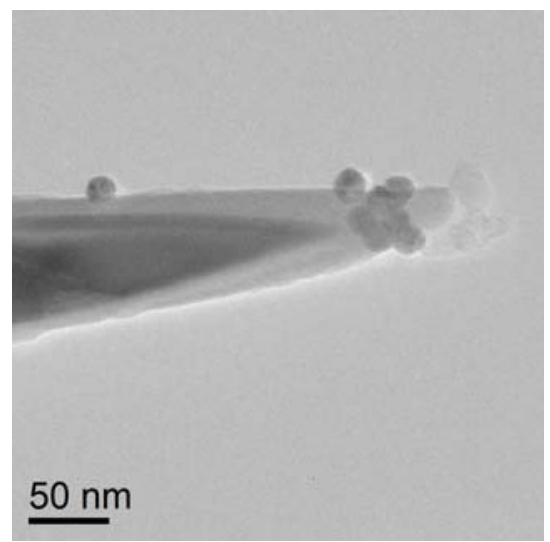

(a)

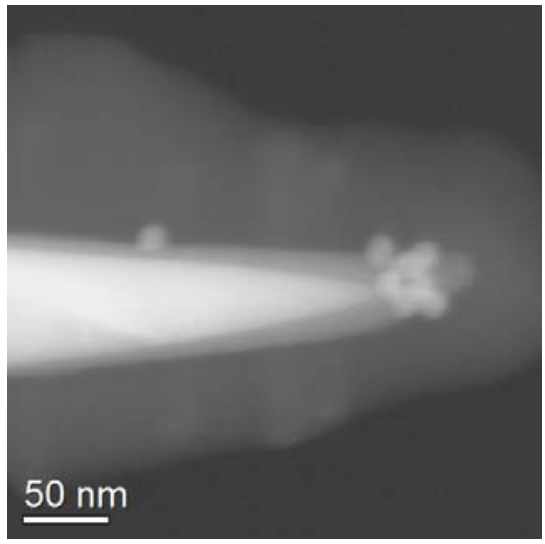

(b)

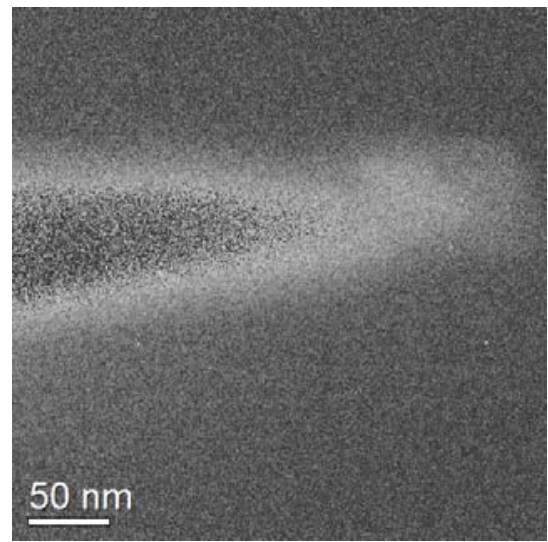

(c)

Fig.1 One typical tungsten tip (at $0^{\circ}$ tilt angle) with 20 -nm gold particles attached to its surface; BF image (a), ADF STEM image (b) and oxygen mapping by EFTEM (c).

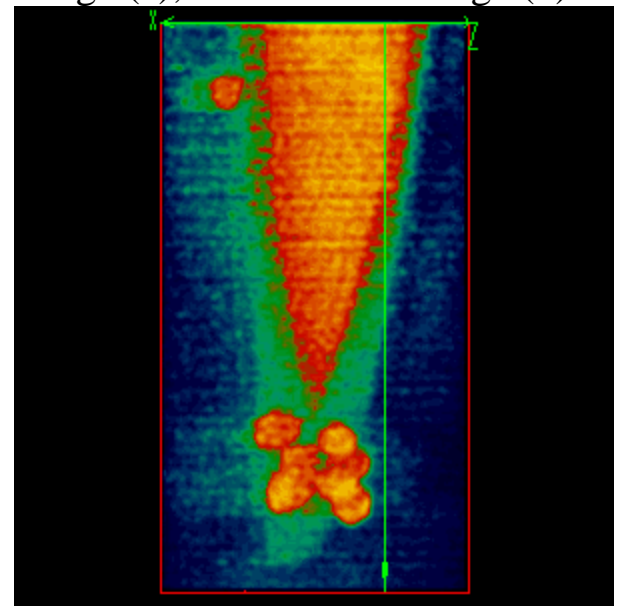

(a)

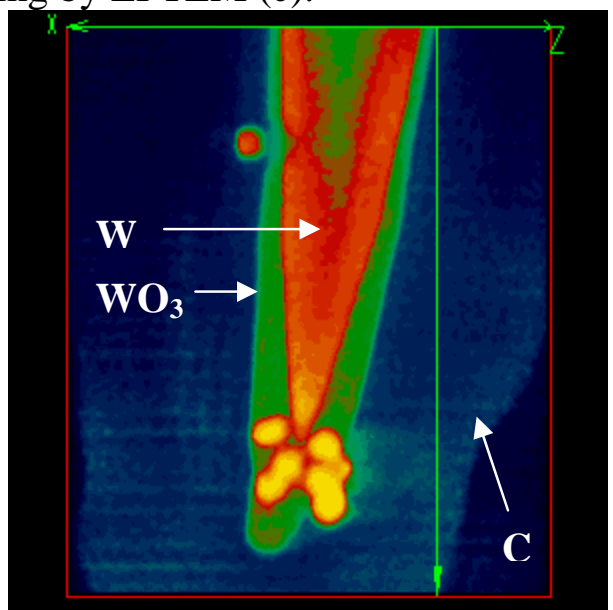

(b)

Fig.2 Tomograms by BF (a) and ADF-STEM (b); Tungsten oxide is shown in green colour; tungsten and gold particles are in red and yellow colour; carbon contamination is in blue.

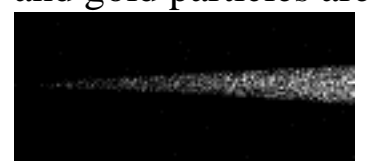

(a)

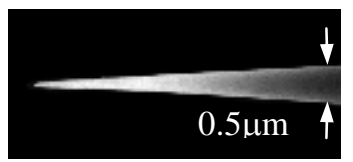

(b)

Fig.3 Tungsten thickness mapping by EDX (a) and ADF (b). Image of another tungsten tip than Fig 2. 